\title{
INCONSTITUCIONALIDAD, LEGALIDAD Y ORDEN JURÍDICO
}

\author{
Pablo Martín Perot \\ Universidad Nacional de Mar del Plata
}

\section{Introducción}

Corrientemente se concibe a la constitución como un conjunto de normas fundamentales. Ello es así ya que en los sistemas democráticos contemporáneos ella juega un papel central, tanto en el diseño institucional como en la protección de los individuos frente al poder constituido. En este contexto, tachar de "inconstitucional" a una norma implica una carga emotiva desfavorable, que la descalifica como norma, con la pretensión de que ella no sea utilizada para justificar decisiones institucionales. Como en la práctica jurídica este término está impregnado de una fuerte carga emotiva y de una constante utilización ideológica, resulta extremadamente complejo hacer una reconstrucción de su significado que recoja los diferentes usos. Por ello, uno de los desafíos de la teoría jurídica es brindar una caracterización de la noción de "inconstitucionalidad", independiente de su utilización ideológica, a partir de la cual se pueda dar cuenta de las consecuencias que se derivan de calificar a una norma de tal manera.

En el trabajo "Inconstitucionalidad y derogación" (en adelante "I. y D.") se realizan dos pasos necesarios para lograr este objetivo. En primer lugar, se presenta la disolución del "problema" de la existencia de las normas inconstitucionales. Esto es importante dado que con frecuencia se plantea la cuestión de si existen -y en tal caso en qué sentido existen- las normas inconstitucionales, como algo que es necesario elucidar previamente para poder explicar si la norma es válida en algún sentido. En segundo lugar, se introduce la distinción entre orden jurídico depurado y orden jurídico no depurado, a partir de interpretaciones diversas del funcionamiento del criterio jerárquico lex superior (preservando o restableciendo la consistencia). Esta distinción resulta relevante, ya que la noción de "inconstitucionalidad" funciona de manera diferente en relación con cada reconstrucción. Es decir, que la inconstitucionalidad de una norma trae aparejadas consecuencias diversas en cada uno de los modelos de orden jurídico. 
En el presente trabajo se intentará efectuar algunas precisiones conceptuales respecto de la noción de "inconstitucionalidad", para luego ofrecer reconstrucciones diversas del criterio de legalidad que presupone cada modelo de orden jurídico. Para finalizar, se planteará un interrogante que permite marcar cierta relación entre el orden jurídico depurado y los presupuestos del positivismo incluyente.

\section{Inconstitucionalidad y existencia de las normas}

Como se dijo, uno de los objetivos que se plantean en "I. y D." es el de plantear el tradicional "problema" de la existencia de las normas inconstitucionales como un seudo-problema a fin de disolverlo. Para lograr tal objetivo, se analiza "...en qué sentido puede decirse que una norma inconstitucional existe, si por ella se entiende una formulación normativa no interpretada [o interpretada] que ha sido creada irregularmente por falta de satisfacción de los requisitos impuestos para ello por normas contenidas en la constitución.", para concluir que "...ya sea que se conciba a las normas como formulaciones interpretadas o no interpretadas, la existencia de una norma no depende de su constitucionalidad (formal o material). Esta última característica puede sí determinar que se la considere o no parte del derecho y puede repercutir sobre los efectos que se le reconozcan, pero esas son cuestiones diferentes."

Esta conclusión se alcanza de la siguiente manera. En primer lugar, se toma a la noción de "inconstitucionalidad" como una especie del género "invalidez". La "validez" se define como regularidad de la creación normativa. En este sentido, se resalta que no debe identificarse validez con pertenencia, por cuanto hay normas en los sistemas jurídicos que pueden pertenecer a él por criterios diferentes al de su creación regular (por ejemplo, las normas que se denominan "soberanas", "no derivadas" o "independientes"). Así, la invalidez es entendida como la creación irregular de una norma, y se reserva el término "inconstitucionalidad" para las normas cuya "(...) invalidez resultase del incumplimiento de los requisitos impuestos por normas contenidas en la constitución (las normas de la máxima jerarquía) para la creación de otras normas." Se distingue, como es corriente, entre "inconstitucionalidad formal" (IF) e "inconstitucionalidad material" (IM), ya que las exigencias de las normas jerárquicamente superiores se refieren tanto al órgano competente para la promulgación de otras normas, al procedimiento a seguir para ello, como al contenido que éstas pueden poseer. De esta manera, se predica de una norma IF cuando el acto de promulgación fue realizado por una autoridad incompetente o por un procedimiento impropio de acuerdo con la constitución, e IM cuando 
el resultado o contenido del acto de promulgación (la norma) es lógicamente incompatible con las normas constitucionales.

En segundo lugar, se utilizan las distinciones conceptuales propuestas por Ricardo CARACCIOLO entre conceptos o concepciones de las normas, dimensiones o modos de existencia de las normas y propiedades que se pueden atribuir a ellas. Estas distinciones sirven para afirmar que la elección de cierto concepto de norma condiciona la adopción de una de las dos posibles dimensiones de existencia: empírica o abstracta; y además, que el asumir cierta ontología condiciona las propiedades que pueden atribuirse a las normas (validez, aplicabilidad, eficacia, obligatoriedad, etc). ${ }^{1}$ El atributo de "inconstitucional" no es una excepción y, por ello, el sentido en que pueda decirse que una norma es inconstitucional está condicionado por la ontología asumida.

En tercer lugar, se consideran dos concepciones de las normas: la que las entiende como formulaciones no interpretadas y la que las concibe como formulaciones interpretadas. Si se considera que la voz "norma" se refiere a formulaciones no interpretadas, es necesario distinguir entre formulaciones-caso y formulación-tipo a fin de saber qué ontología de las normas condiciona dicho concepto. Si se las entiende como formulaciones-caso, es decir, como objetos físicos (en el caso del lenguaje escrito) o fenómenos físicos (en el caso del lenguaje oral), ellas existen como entidades concretas localizadas en el espacio-tiempo por el sólo hecho de haber sido formuladas. Si se las considera como formulaciones-tipo, esto es, como arquetipos, modelos o formas lingüísticas ideales, entonces existen como entidades abstractas fuera del espacio-tiempo. También, respecto de la concepción de las normas como formulaciones interpretadas, es necesario distinguir entre formulaciones-caso y formulacionestipo, para saber qué ontología se asume con tal concepción. Si las normas se entienden como formulaciones-caso significativas, su existencia sería concreta y dependería de su mera formulación, en cambio, si se las concibe como formulaciones-tipo interpretadas las normas existirían como entidades abstractas.

Por todo ello se concluye que la inconstitucionalidad no puede repercutir sobre la existencia de las normas, porque: o bien su existencia depende de la mera formulación en el lenguaje por ser entidades empíricas, o bien su existencia no depende de la realización de hecho alguno por ser entidades abstractas, de modo que la existencia de las normas resultaría, en ambos casos, independiente de su "creación regular".

En "I. y D.", con justas razones -que se comparten-, se descarta la 
posibilidad de concebir a las normas como formulaciones-caso (interpretadas o no) por acarrear consecuencias que la hacen insostenible. La más importante de estas consecuencias es que no resulta posible desde esta concepción una reconstrucción sistemática del derecho, lo cual impide brindar una caracterización adecuada de la inconstitucionalidad que presupone tales relaciones sistemáticas. Por ello, de aquí en adelante y salvo aclaración en contrario, se utilizará la voz "norma" para hacer referencia a formulaciones-tipo interpretadas.

Sin embargo, si se disuelve el problema de la manera descripta, resulta conveniente efectuar dos precisiones. En primer lugar, parece criticable definir "inconstitucionalidad" en función de la noción de "creación" dado que, si no se aclara que se está haciendo un uso metafórico de la expresión, ello puede volver a introducir las confusiones que se pretenden evitar con la disolución del problema. Como sostiene Caracciolo, "Usualmente se caracteriza el acto de promulgación como la "creación" de una norma. No obstante, esa manera de hablar sólo puede tratarse de una metáfora. Porque si las normas son entidades intensionales carece de sentido decir que son "creadas" por actos, y si no existen tales entidades no hay nada que pueda ser "creado" por actos semejantes..."

En segundo lugar, resulta importante destacar la diferencia que media entre el acto de promulgación y la norma promulgada que es el resultado o contenido de dicho acto. De acuerdo con CARACCIOlO, el enunciado " $x$ promulga $N$ " supone que hay que distinguir la norma mencionada " $N$ " del hecho que describe tal enunciado. Esto es importante porque en esta distinción se basa la posibilidad de establecer relaciones entre eventos y normas, relaciones que pueden utilizarse como criterios empíricos para construir conjuntos de tales entidades. ${ }^{3}$ Por ello, en lo que sigue se entenderá por "promulgación" al acto o evento por el cual una autoridad da a conocer una formulación normativa. ${ }^{4}$

A esta altura, es posible plantear la siguiente cuestión: ¿qué influencia tiene la concepción de las normas que se adopte respecto de la posibilidad de aplicar el predicado "inconstitucional"? Con lo dicho hasta aquí, sólo se pueden afirmar dos cosas. En primer lugar, que IF se refiere de manera originaria al acto de promulgación, y sólo de manera derivada puede calificar a la norma que es el resultado o contenido de dicho acto. En segundo lugar, que IM sólo puede predicarse en sentido propio de formulaciones interpretadas.

Puede predicarse IF de una norma, en un sentido derivado, cuando el acto de promulgación de la formulación normativa a partir del cual se la identifica ha sido irregular con relación a las condiciones exigidas por la constitución para 
la realización de ese acto. Sin embargo, esto no surge claramente de "I. y D." porque allí sostienen los autores que IF se refiere en sentido originario a las formulaciones no interpretadas, y sólo en sentido derivado a las formulaciones interpretadas. ${ }^{5}$ En realidad, el uso en ambos casos es derivado, ya que en ambos se afirma que el acto de promulgación ha sido irregular y, por ello, se califica de inconstitucional a las normas que se identifiquen a partir de ese acto, se considere a éstas como formulaciones interpretadas o como formulaciones no interpretadas. Es decir, que IF se puede predicar de las normas sólo en un sentido derivado, y esto es independiente de la concepción que se adopte a su respecto.

En cambio, con relación a IM sí tiene influencia la concepción de las normas que se asuma. Como se afirma en el trabajo, este predicado sólo puede atribuirse en sentido propio a formulaciones interpretadas, y sólo en un sentido derivado y algo impropio a formulaciones no interpretadas. Esto es así porque entre tales entidades no pueden existir relaciones lógicas.

Lo dicho en los párrafos anteriores pone de manifiesto un inconveniente importante a la hora de reconstruir la noción "inconstitucionalidad": que de acuerdo con el uso que se hace de tal expresión, el campo de su significado es heterogéneo. Esa heterogeneidad está dada por el hecho de que el término se utiliza como atributo de entidades diferentes. La IF predica que un evento (acto de promulgación) no satisface ciertas exigencias constitucionales, en cambio la IM atribuye la propiedad de ser incompatible con la constitución a entidades abstractas (normas). Si bien en la práctica ésta heterogeneidad semántica se oculta detrás de cierta homogeneidad pragmática -porque en definitiva tanto IF como IM se utilizan con fines similares: descartar a una norma como posible justificación institucional-, el hecho de que se emplee para calificar entidades diferentes pone en tela de juicio la posibilidad de dar una caracterización general que comprenda ambos usos.

\section{Inconstitucionalidad y legalidad}

Como afirma Hans KeLSEN, una de las características salientes del derecho es la de regular su propia creación, en el sentido de que son las normas jurídicas las que regulan la producción de otras normas jurídicas. Sin embargo, a partir de este dato, surgen diversas posibilidades teóricas a la hora de dar cuenta de tales relaciones genéticas. La noción de "inconstitucionalidad" está ligada de forma estrecha al modelo de reconstrucción de estas relaciones entre las normas, porque las consecuencias de afirmar la inconstitucionalidad de una norma pueden variar en cada una de estas reconstrucciones. 
Si se considera, desde un punto de vista estático, un sistema jurídico que sólo tiene como fuente de derecho a la legislación, generalmente se acepta que tres criterios son suficientes como reglas de admisión de normas en el sistema: extensionalidad (Ce) -el conjunto de normas $C$ pertenece a $\alpha$-; legalidad $(\mathrm{Cl})$-si hay una norma $p$ que autoriza a una autoridad $x$ para dictar la norma $q$, y $p$ pertenece a $\alpha$, y $x$ dicta $q$, entonces $q$ pertenece a $\alpha$ - y deducibilidad (Cd) -todas las normas que son consecuencias lógicas de las normas que pertenecen a $\alpha$ también pertenecen a $\alpha-.^{6}$ Los tres criterios funcionan como un esquema definicional de "sistema jurídico" y sirven para identificar las normas que pertenecen a los diferentes sistemas. Por Ce se puede establecer por enumeración el conjunto de normas que se consideran de máxima jerarquía o soberanas del sistema y definen a cada sistema en particular. A este conjunto normalmente se lo designa como "constitución". Por medio de $\mathrm{Cl}$, se recurre a un criterio empírico de pertenencia que asume la ocurrencia de uno o varios hechos como indicador para integrar un conjunto de normas. ${ }^{7}$ Y Cd no sería más que una consecuencia de concebir a los sistemas jurídicos como sistemas deductivos.

Entre las normas soberanas identificadas mediante Ce debe haber por lo menos una que atribuya competencia a cierta autoridad para dictar nuevas normas. De acuerdo con AlchourRón y BuLYGIN, ${ }^{8} \mathrm{Cl}$ es una regla conceptual que hace referencia a normas de competencia del sistema. Entre ellas se encuentra la o las reglas de competencia contenidas en las normas constitucionales. Como las exigencias de las normas constitucionales pueden referirse al órgano competente para la promulgación de la norma, al procedimiento a seguir para ello y al contenido de la norma en cuestión, $\mathrm{Cl}$ pude reconstruirse de diversas manera de acuerdo con la relevancia que se le otorgue a cada una de ellas. En una primera lectura se consideran relevantes sólo las exigencias referidas al procedimiento y al órgano competente; en una segunda, además, se toman en cuenta las que se refieren al contenido de las normas promulgadas. Así, podríamos diferenciar: ${ }^{9}$

$\mathrm{Cl}_{1}$ : Si hay una norma $p$ que autoriza a una autoridad $x$ para dictar la norma $q$ a través del procedimiento $y$, y $p$ pertenece a $\alpha, y x$ dicta $q$ a través del procedimiento $y$, entonces $q$ pertenece al sistema $\alpha$.

$\mathrm{Cl}_{2}$ : Si hay una norma $p$ que autoriza a una autoridad $x$ para dictar la norma $q$ a través del procedimiento $y$, y $p$ pertenece a $\alpha$, y $x$ dicta $q$ a través del procedimiento $y$, entonces $q$ pertenece al sistema $\alpha$, si y solo si $q$ no es incompatible con las consecuencias lógicas del subconjunto $C$ (constitución) de $\alpha$. 
La diferencia entre las dos lecturas, $\mathrm{Cl}_{1}$ y $\mathrm{Cl}_{2}$, es que en la segunda aparece una restricción que establece que no se deben considerar parte del sistema a las normas incompatibles con la constitución. De esta manera, las consecuencias de predicar la inconstitucionalidad de una norma pueden variar en cada una de las reconstrucciones de $\mathrm{Cl}$. Resulta claro que predicar IF de una norma implica afirmar -de manera derivada o impropia- que ella no satisface el criterio empírico de pertenencia que establece $\mathrm{Cl}$ en cualquiera de las dos reconstrucciones posibles. Por lo tanto, dicha norma no se puede considerar perteneciente al sistema en virtud de tal criterio. Más complejo resulta marcar la relación entre $I \mathrm{M} \mathrm{y} \mathrm{Cl}$. De acuerdo con $\mathrm{Cl}_{1}$, las normas que son materialmente inconstitucionales pertenecen al sistema, mientras que de acuerdo con $\mathrm{Cl}_{2}$, tales normas no pertenecen al sistema al no satisfacer la última restricción en él contenida. De esta manera, los sistemas jurídicos reconstruidos a partir de $\mathrm{Cl}_{2}$ no pueden, por definición, contener contradicciones entre normas infraconstitucionales y normas constitucionales.

\section{Legalidad y competencia}

Hasta aquí se ha obviado una cuestión importante: la caracterización de las reglas de competencia. Como se dijo, $\mathrm{Cl}$ es una regla conceptual que hace referencia a normas de competencia. Por lo tanto, la reconstrucción que se haga de tal criterio depende de la caracterización de éste problemático tipo de normas. En las reconstrucciones anteriores se asumió que la noción de competencia se vincula con normas permisivas. Sin embargo, es posible concebir a tales normas de manera diferente. Si se considera a la atribución de competencia como un fenómeno complejo, entonces -como sostiene Jorge RoDríGuEz- se requiere por lo menos de una regla conceptual que establezca las condiciones para el surgimiento de una instancia válida de cierto predicado jurídico (contrato, matrimonio, testamento, ley, sentencia, etc.) y de una prescripción condicional general que establezca los efectos jurídicos de todas las instancias válidas del predicado jurídico en cuestión. ${ }^{10}$ De acuerdo con esta caracterización de la atribución de competencia puede sugerirse la siguiente reconstrucción de $\mathrm{Cl}$ :

$\mathrm{Cl}^{\prime}$ : Si una norma $p$, que define el predicado jurídico $L$ exigiendo la satisfacción conjunta de las condiciones $C_{1^{\prime}} C_{2^{\prime}}, \ldots, C_{n^{\prime}}$ pertenece a $\alpha$, y la norma $q$ satisface $C_{1}, C_{2}, \ldots, C_{n^{\prime}}$ entonces $q$ pertenece a $\alpha$.

$\mathrm{Cl}^{\prime}$ sigue siendo una regla conceptual, pero en esta interpretación de las normas de competencia, no hace referencia a una norma permisiva sino a otra regla conceptual que pertenece al sistema y define un predicado jurídico $L$ (ley, 
por ejemplo). Puede señalarse como ventaja de la reconstrucción de $\mathrm{Cl}^{\prime}$ que se refiere a cierta clase de normas (la clase de normas que satisfacen $L$ ) y no, como sucedía en $\mathrm{Cl}$, a una norma determinada (la norma $q$ ). Este parece un modo más adecuado de reconstrucción de las normas de competencia, dado que sólo en casos excepcionales y en niveles inferiores de la jerarquía normativa, la atribución de competencia funciona indicando una norma determinada y no una cierta clase de normas. ${ }^{11}$ En la Constitución Nacional argentina, por ejemplo, los artículos 77 al 84 fijan las condiciones para el dictado de leyes nacionales válidas, por lo que esos artículos definen en este sentido "ley válida". Por otro lado, $\mathrm{Cl}^{\prime}$ omite la referencia a la prescripción condicional que forma parte de la atribución de competencia. Ello porque, si bien tal prescripción es necesaria para establecer los efectos jurídicos de las instancias válidas del predicado jurídico " $L$ " -y, por lo tanto, para afirmar que existe atribución de competencia-, resulta prescindible a la hora de establecer la pertenencia de las normas al sistema. ${ }^{12}$ En este sentido pueden tomarse las palabras de Rodríguez: "...En eso consiste la atribución de competencia y los límites de la competencia: no en permitir dictar normas sino en reputar pertenecientes al sistema a las normas dictadas por ciertas autoridades bajo ciertas condiciones...".(cf. RodríGuez 1999).

Como las condiciones que especifica la regla conceptual $\left(C_{1}, C_{2}, \ldots, C_{n}\right)$ generalmente se refieren al procedimiento, al órgano competente y al contenido de las normas, para recoger a partir de $\mathrm{Cl}^{\prime}$ la diferencia que existía entre $\mathrm{Cl}_{1}$ y $\mathrm{Cl}_{2}$, habría que distinguir entre dos formas alternativas de reconstruir esa regla conceptual. En una primera versión, asimilable a $\mathrm{Cl}_{1}$, la regla conceptual sólo definiría el predicado jurídico teniendo en cuenta el procedimiento y la autoridad competente. En cambio, en una segunda versión, asimilable a $\mathrm{Cl}_{2}$, la regla conceptual también contendría la exigencia de que el contenido de las diferentes instancias del predicado jurídico no sea incompatible con las normas jerárquicamente superiores. De esta manera, el conjunto de disposiciones constitucionales (en especial, de aquellas que confieren derechos) funcionan como limitaciones o restricciones al campo de aplicación del predicado jurídico "ley válida", al no considerar válidas a aquellas normas que sean incompatibles con las normas constitucionales. Sólo en éste último caso, al igual que ocurría con $\mathrm{Cl}_{2}$, predicar IM de una norma implica que ella, por ser un caso que no puede ser subsumido en el predicado jurídico $L$, no pertenece al sistema jurídico en cuestión. Entonces, también por definición, los sistemas jurídicos reconstruidos a partir de esta lectura de $\mathrm{Cl}^{\prime}$ no pueden contener contradicciones entre normas infraconstitucionales y normas de jerarquía constitucional. ${ }^{13}$

Todavía, sin embargo, es conveniente hacer una importante precisión. De 
acuerdo con Jorge RodRíGuez, la introducción de Cl como criterio de pertenencia de normas a un sistema jurídico resulta altamente discutible. En contra del profesor BULYGIN, concuerda plenamente con la opinión de CARACCIOLO en torno a que $\mathrm{Cl}$ no suministra en verdad condiciones de pertenencia de normas a un sistema, sino que define la pertenencia de conjuntos de normas al orden jurídico. ${ }^{14}$ Así, como en sentido estricto $\mathrm{Cl}$ formaría parte del esquema definicional de "orden jurídico" y no de "sistema jurídico", las diferencias marcadas en las diferentes reconstrucciones de $\mathrm{Cl}$ y sus consecuencias respecto de la inconstitucionalidad deberían basarse en un análisis dinámico y no estático del derecho.

En "I. y D." se introduce la distinción entre orden jurídico depurado (OJD) y orden jurídico no depurado (OJND), que constituye una de las contribuciones más originales del trabajo. Desde un punto de vista dinámico, los criterios de preferencias entre normas "...pueden operar de dos maneras distintas: 'ex ante', preservando la consistencia e impidiendo que el acto de promulgación de una norma produzca un nuevo sistema en la secuencia si esa norma es lógicamente incompatible con alguna de las preexistentes, o 'ex post', restableciendo la consistencia una vez que se ha admitido el ingreso de un sistema inconsistente en el orden jurídico...". Tomando en cuenta esta distinción respecto del criterio de lex superior, se definen en el trabajo las nociones de OJD y OJND. Las diferencias entre estos dos modelos alternativos se fundamentan en que ambos suponen reconstrucciones diferentes y excluyentes de $\mathrm{Cl}$. En este sentido puede entenderse la siguiente afirmación del trabajo citado: "En la primera reconstrucción -el orden jurídico depurado- una de las condiciones de legalidad que debe satisfacer un acto de promulgación para permitir asociar un nuevo sistema al orden jurídico es que la norma o conjunto de normas promulgadas no resulten incompatibles con normas emanadas de un órgano jerárquicamente superior. Se interpretaría así a esta condición como conceptualmente ligada a la noción de competencia, en el sentido que un órgano productor de normas actuaría fuera de su competencia si dictara una norma lógicamente incompatible con otra dictada por un órgano jerárquicamente superior, al menos en la medida de la incompatibilidad...". De acuerdo con la cita, parecería que el modelo OJND asume la primera versión de $\mathrm{Cl}^{\prime}$ (asimilable a $\mathrm{Cl}_{1}$ ), en la cual la regla conceptual sólo definiría el predicado jurídico teniendo en cuenta el procedimiento y la autoridad competente. En cambio, el modelo OJD parece asumir la segunda versión de $\mathrm{Cl}^{\prime}$ (asimilable a $\mathrm{Cl}_{2}$ ), donde la regla conceptual contendría además la exigencia de que el contenido de las diferentes instancias del predicado jurídico no sea incompatible con las normas de jerarquía constitucional. Estas dos posibles versiones de $\mathrm{Cl}^{\prime}$, entonces, dan lugar desde un punto de vista dinámico a las dos posibles reconstrucciones del orden jurídico. 


\section{5. ¿Dos modelos teóricos de reconstrucción del orden jurídico?}

Los autores parecen considerar que los modelos del OJD y del OJND constituyen dos esquemas teóricos diversos a partir de los cuales se puede reconstruir cualquier orden positivo. No obstante, a partir de la reconstrucción de $\mathrm{Cl}^{\prime}$ que cada uno supone, esta apreciación puede ponerse en duda: ¿está condicionada la elección de una de las diferentes reconstrucciones del orden jurídico por el contenido de las normas de los diferentes ordenamientos positivos o depende sólo de consideraciones teóricas? El problema es el siguiente. Como se vio, la distinción entre OJD y OJND se basaba en una diferente reconstrucción de $\mathrm{Cl}$. Una de las maneras de mostrar esta diferencia es hacer una lectura diversa de la regla de competencia a la que hace referencia $\mathrm{Cl}^{\prime}$, una que toma como condición de validez de las normas la compatibilidad entre ellas y las disposiciones constitucionales, y otra que no considera tal condición. Ahora bien, si la elección de alguna de estas diversas lecturas depende de lo que establecen las normas constitucionales que confieren competencia, entonces también la elección del modelo de reconstrucción del orden jurídico está condicionada por el contenido contingente de tales normas. En definitiva, si se acepta esto último, parece ser que OJD y OJND no son dos reconstrucciones posibles de cualquier ordenamiento positivo, sino dos reconstrucciones mutuamente excluyentes de ordenamientos positivos con diferentes contenido (i.e., con reglas constitucionales de competencia que establecen diferentes exigencias).

El problema resulta relevante porque está conectado con una de las críticas que con mayor fuerza se han alzado en contra del positivismo jurídico, y que BAYÓN denomina "argumento del contraste con la práctica". Este argumento afirma que nuestras prácticas jurídicas efectivas, que reiteradamente recurren a la argumentación moral para identificar y aplicar el derecho, desmienten el núcleo definitorio del positivismo jurídico: la tesis de las fuentes sociales del derecho (i.e., que la existencia y contenido del derecho dependen exclusivamente de hechos sociales, por lo que su identificación es posible sin recurrir a argumentos morales). Lo que discuten los autores que sostienen este argumento no es que la tesis de las fuentes sociales sea rechazable por razones conceptuales, sino que resulta descriptivamente inadecuada cuando se la confronta con la práctica jurídica real. En palabras de BAYÓN: "... Esto es, no discuten que la realidad de un sistema jurídico 'podría' ser de un tipo tal que la tesis de las fuentes sociales resultase verdadera: lo que sostiene es que, como cuestión de hecho, nuestros sistemas jurídicos simplemente no son así. "15 
Dos formas de rechazo de este argumento son analizadas por BAYÓN. La primera afirma que la tesis de las fuentes sociales es una tesis conceptual y por lo tanto no puede ser refutada por la práctica. Esta posición es la sustentada por dos corrientes alternativas que BAYÓN denomina "positivismo simple" y "positivismo excluyente". La segunda, en cambio, acepta la tesis de las fuente sociales como una tesis empírica e intenta reformularla de manera que resulte inmune ante las diferentes posibilidades de configuración de nuestras prácticas. ${ }^{16}$ Esta última estrategia es la desarrollada por el denominado "positivismo incluyente" o "incorporacionismo", y es la que aquí interesa. Más específicamente, esta corriente sostiene que: "...para evitar el alegado contraste entre la teoría jurídica positivista y lo que parece ser un rasgo de nuestras prácticas jurídicas (que el razonamiento moral ocupa de algún modo un lugar entre ellas) (...) afirma por tanto que, en lo que concierne a sistemas jurídicos como los nuestros, hay (al menos algunas) normas morales que se han convertido auténticamente en parte del derecho en virtud de su valor, de su contenido, pero que ello es así sólo por el hecho (contingente) de que nuestra práctica jurídica incluye la convención de reconocerlas como tal."17 En definitiva, lo que el positivismo incluyente sostiene es que en ciertos sistemas jurídicos la existencia y contenido del derecho puede depender de valoraciones y principios morales, como sería el caso de las democracias contemporáneas en las que la validez de las leyes depende de su conformidad con ciertos principios morales básicos tutelados por sus constituciones.

OJD parece recoger, en parte, la misma idea. Ello es así porque, de acuerdo con este modelo, es necesaria la evaluación del contenido de las normas para saber si ellas forman parte del derecho. Si a esto se le suma el hecho de que las democracias constitucionales contemporáneas tutelan ciertos principios morales básicos en sus constituciones, entonces la pertenencia de algunas normas a dichos sistemas jurídicos dependería de la evaluación moral de su contenido. Sin embargo, la asimilación no es tan sencilla porque para que OJD responda a las pretensiones del incorporacionismo deberían considerarse dos cuestiones adicionales. En primer lugar, un positivista incluyente debería afirmar que en ciertos casos la corrección moral de una norma es condición necesaria y a la vez suficiente de la validez de la norma, para que su posición pueda distinguirse de la de un positivista excluyente. ${ }^{18}$ Sin embargo en OJD la restricción respecto del contenido de las normas que se identifican a partir de las formulaciones promulgadas funciona como una condición necesaria pero no suficiente de la validez de las normas. En segundo lugar, como consecuencia de interpretar a la tesis de las fuentes sociales como una tesis empírica, el incorporacionismo debería entender que reconstruir un derecho positivo a partir de OJD o OJND depende 
de lo que digan sus normas. Y esta es una cuestión que, como ya se dijo, queda pendiente de respuesta al menos de conformidad con la presentación que los autores hacen de la distinción. 


\title{
Notas
}

\author{
${ }^{1}$ Cf. Caracciolo 1997. \\ ${ }^{2}$ Caracciolo 1997:170, nota 22. \\ ${ }^{3}$ Cf. Caracciolo 1997:171. \\ ${ }^{4}$ Cf. von WRIGHT 1963:131. \\ ${ }^{5}$ En "I. y D." por ejemplo se afirma que: "...validez formal y validez material se predican al menos
} en sentido originario de entidades diferentes (la primera, de formulaciones no interpretadas; la segunda, de formulaciones interpretadas)...". Es cierto que validez formal y validez material se predican de entidades diferentes, pero no que esas entidades sean una formulación no interpretada y una formulación interpretada. La diferencia radica en que la validez formal se predica del acto de promulgación mientras que la validez material se predica de la interpretación acordada al resultado de dicho acto. Lo que sucede, es que la validez material sólo puede predicarse en sentido propio de entidades entre las que pueden existir relaciones lógicas, es decir entre formulaciones interpretadas y no entre formulaciones no interpretadas.

${ }^{6}$ Cfr. Alchourrón-Bulygin 1971:120 y AlChOurRón-Bulygin 1979:73-76.

7 Cfr. Caracciolo 1997: 175.

${ }^{8}$ AlCHOURRÓN-Bulygin 1971:121.

${ }^{9} \mathrm{~A}$ fin de simplificar la exposición, no se tomarán en cuenta aquí las salvedades introducidas en el punto anterior. De hacerlo, la formalización de $\mathrm{Cl}_{1}$, por ejemplo, debería ser la siguiente: $\mathrm{Cl}_{1}$ : Si hay una norma $p$ que autoriza a una autoridad $x$ para dictar la formulación normativa $f$ a través del procedimiento $y$, y $p$ pertenece a $\alpha, y x$ dicta $f$ a través del procedimiento $y, y$ la norma $q$ se identifica a partir de la formulación $f$, entonces $q$ pertenece al sistema $\alpha$.

${ }^{10}$ Cf. RodríGuez 1999.

${ }^{11}$ Esta corrección fue sugerida por una crítica similar efectuada por respecto de la aplicabilidad (cf. RODRíGuez 1999). Una disposición como la contenida en el artículo 24 de la Constitución Argentina, que establece que "El Congreso promoverá... el establecimiento del juicio por jurados" parece entenderse mejor si se considera que está otorgando competencia para promulgar una clase de normas (la clase de normas que establecen el juicio por jurados) y no una norma determinada, ya que el establecimiento del juicio por jurados puede hacerse a través de diferentes normas que pertenecen a la misma clase.

${ }^{12}$ Podría pensarse que si las prescripciones condicionales que establecen los efectos de las instancias válidas de los predicados jurídicos son prescindibles para afirmar la pertenencia de las normas al sistema, entonces lo son también para caracterizar la atribución de competencia; de modo que para ello bastaría con la regla conceptual que define a tales predicados. Sin embargo, esto dejaría de lado algo que es muy común en la práctica: que los predicados jurídicos se definen para que cada una de sus instancias válidas produzcan efectos jurídicos. En el caso de que una autoridad defina un predicado y no establezca ningún efecto jurídico, quizás sería correcto afirmar que las instancias válidas de cada predicado pertenecen al sistema, pero que en tal caso no se atribuyó competencia.

13 RoDríguez expresa esta diferencia de la siguiente manera: "Si una autoridad normativa promulga una norma que contradice otra dictada por una autoridad de jerarquía superior, puede considerarse indistintamente: a) que ella no ingresa al sistema por haber sido derogada anticipadamente por la autoridad superior, en cuyo caso, no contradecir las normas dictadas por autoridades jerárquicamente superiores constituiría una condición para la promulgación de normas válidas y un límite a la competencia de la autoridad inferior; o b) que ella ingresa al sistema, generándose así una contradicción, para cuya eliminación habrá que utilizar algún criterio jerárquico (como el de lex superior). En la primera interpretación, se incorpora al sistema una jerarquía entre sus normas; en la segunda, las jerarquías entre normas resultan extrasistemáticas. Si en el texto se dio preferencia a la primer opción es porque ella parece ajustarse mejor a las intuiciones de los juristas." (cf. RodRíGuEz 1999). 
${ }^{14}$ Cfr. Caracciolo 1988; Bulygin 1991; RodríGuez 1999.

${ }^{15}$ BAYÓN 2000:1-5.

${ }^{16}$ BAYÓN 2000: 6.

17 BAYÓN 2000: 21.

${ }^{18}$ BAYÓN 2000: 25-26.

\section{Bibliografía}

Alchourrón, C. y BulyGin, E., 1971, Introducción a la metodología de las ciencias jurídicas y sociales, Buenos Aires, Astrea, 1975, título original: Normative Systems, Viena, Springer-Verlag, traducido por los autores.

Alchourrón, C. y Bulrgin, E., 1976, "Sobre el concepto de orden jurídico", en Crítica, Vol. VIII, No 23.

Bayón, J. C., 2000, "Derecho, convencionalismo y controversia", trabajo inédito, obtenido por gentileza del autor.

ButrGin, E., 1991, "Algunas consideraciones sobre los sistemas jurídicos", en Doxa - Cuadernos de Filosofía del Derecho 10: 257-279.

Caracciolo, R., 1988, El Sistema Jurídico. Problemas actuales, Madrid, Centro de Estudios Constitucionales.

CARACCIOlo, R., 1997, "Existencia de normas", en Isonomía, número 7.

Kelsen, H., 1960, Teoría Pura del Derecho (segunda edición), México, UNAM, 1979, título original: Reine Rechtslehre, Viena, traducido por R. Vernengo.

Orunesu, C., Rodríguez, J. y Sucar, G., "Inconstitucionalidad y derogación", trabajo inédito, obtenido por gentileza de los autores.

RodríGuez, J., 1999, Lógica de los sistemas jurídicos, tesis doctoral inédita, obtenida por gentileza del autor.

von WriGHT, G. 1963 Norma y acción. Una investigación lógica, Madrid, Tecnos, 1970, título original: Norm and Action. A Logical Inquiry, Londres, Routledge \& Kegan Paul, 1963, traducido por P. GARCíA FERRERO. 